\title{
AVALIAÇÃO DA VERTICAL VISUAL SUBJETIVA EM INDIVÍDUOS BRASILEIROS NORMAIS
}

\author{
Aline M. Kozoroski Kanashiro', Cristiana Borges Pereira², \\ Fernanda Martins Maia', Milberto Scaff ${ }^{3}$, Egberto Reis Barbosa ${ }^{4}$
}

\begin{abstract}
RESUMO - A função otolítica pode ser avaliada pela Vertical Visual Subjetiva (VVS) que determina a capacidade de um indivíduo julgar se objetos estão na posição vertical na ausência de outras referências visuais. O objetivo deste estudo foi avaliar a VVS em indivíduos brasileiros normais usando um aparelho portátil. As medidas da VVS foram realizadas em 160 indivíduos (16 a 85 anos). O valor médio da VVS foi obtido após dez ajustes. A VVS teve valores médios entre $-2,0^{\circ}$ e $+2,4^{\circ}$ (média $=0,18^{\circ}$, e $D P=0,77^{\circ}$ ). Não houve diferença entre as médias da VVS em relação à idade (teste de Kruskal-Wallis; $p=0,40$ ), mas as faixas etárias maiores tiveram variância maior (teste de Levene; $p=0,016)$. Os valores da VVS encontrados neste estudo foram semelhantes aos registrados na literatura. Não houve diferença nas médias das inclinações da VVS de acordo com a idade, mas foi encontrada maior variância entre indivíduos mais idosos.
\end{abstract}

PALAVRAS-CHAVE: sistema vestibular, visual vertical subjetiva, função otolítica.

\section{Subjective visual vertical evaluation in normal Brazilian subjects}

ABSTRACT - Otolith function can be evaluated by subjective visual vertical (SVV) that determine the capacity of a subject to judge if the objects are on vertical position with absence of any visual reference. The aim of this study was to evaluate the SVV in a sample of normal Brazilian subjects using a portable device. Measurements of SVV were performed in 160 normal subjects (aged from 16 to 85 ). SVV mean value was obtained after ten adjustments. SVV mean values ranged from $-2.0^{\circ}$ to $+2.4^{\circ}$ (mean $=0.18^{\circ}$, and $S D=0.77$ ). Considering all age groups, there was no difference of SVV mean values (Kruskal-Wallis test; $p=0.40$ ), but older groups had a greater variance (Levene test; $p=0.016$ ). SVV values observed in this study are comparable to those described in previous studies. Although there was no difference in mean SVV-inclination according to age, there was a greater variance in older subjects.

KEY WORDS: vestibular system, subjective visual vertical, otolith function.

O sistema vestibular tem a função de estabilização da imagem na retina, manutenção da postura e do equilíbrio, orientação espacial e percepção do movimento, requerendo a integração com os sistemas motor ocular, visual e proprioceptivo'. Os órgãos otolíticos, utrículo e sáculo, fornecem informações ao sistema vestibular sobre movimentos de aceleração linear da cabeça e contribuem para a percepção de orientação espacial, que é a capacidade de um indivíduo perceber a vertical e horizontal gravitacionais ${ }^{2}$. As síndromes vestibulares são diagnosticadas por meio da anamnese e do exame físico dirigidos ao sistema vestibular ${ }^{3}$. Os exames complementares de avaliação da função vestibular colaboram com a confirmação diagnóstica, fornecendo dados e/ou registros que muitas vezes são capazes de quantificar o comprometimento deste sistema, tal como o teste da Vertical Visual Subjetiva (VVS).

Em situações habituais, o julgamento de verticalidade e horizontalidade é realizado principalmente através das informações visuais, pois há, em qualquer ambiente, linhas verticais e horizontais que auxiliam nesta função. Quando as informações visuais são excluídas, por exemplo, em uma sala completamente escura, uma pessoa normal é capaz de julgar se um bastão iluminado está na posição vertical ou horizontal na ausência de qualquer outra aferência visual. Esta capacidade de ajustar o bastão iluminado na posição correta (vertical ou horizontal) depende da informação proveniente do sistema vestibular, através dos órgãos otolíticos ${ }^{4}$. A VVS examina esta capacidade de julgar se objetos estão na posição ver-

Divisão de Clínica Neurológica do Hospital das Clínicas da Faculdade de Medicina da Universidade de São Paulo, São Paulo SP, Brasil (HC-FMUSP): ${ }^{1}$ Pós-Graduanda do Departamento de Neurologia da FMUSP; ${ }^{2}$ Médica responsável pelo Ambulatório de Distúrbios Vestibulares do HC-FMUSP; ${ }^{3}$ Professor-Titular do Departamento de Neurologia da FMUSP; ${ }^{4}$ Docente Livre da FMUSP.

Recebido 4 Agosto 2006, recebido na forma final 1 Dezembro 2006. Aceito 12 Fevereiro 2007. 
tical, ou seja, avalia-se a percepção de verticalidade sem nenhuma referência visual da vertical verdadei$\mathrm{ra}^{5}$. As inclinações entre a vertical percebida pelo indivíduo e a vertical verdadeira são medidas em graus, com uma a média de erro no julgamento da vertical gravitacional entre -2 e +2 graus $^{6}$.

As inclinações da VVS têm o seguinte valor topográfico: (1) em lesões unilaterais do sistema vestibular periférico, o desvio da VVS é para o mesmo lado da lesão (ipsiversivo); (2) nas lesões vestibulares bulbopontinas unilaterais, há um desvio ipsiversivo da VVS; (3) em lesões pontomesencefálicas unilaterais a inclinação da VVS é para o lado oposto ao da lesão, e indica um envolvimento do fascículo longitudinal medial; (4) nas lesões unilaterais do mesencéfalo rostral e núcleo intersticial de Cajal, assim como nas lesões pontomesencefálicas, a VVS tem um desvio na direção oposta à da lesão; (5) em lesões unilaterais do tálamo, os desvios da VVS podem ser tanto ipsiversivos como contraversivos, mas os desvios não mudam em um mesmo paciente e indicam envolvimento da porção posterolateral talâmica; (6) lesões unilaterais do córtex vestibular parieto-insular resultam principalmente em inclinações da VVS contralaterais à lesão ${ }^{7-9}$.

Embora haja estudos na literatura demonstrando a importância do teste da VVS, não há trabalhos que demonstrem quais são os valores normais quando
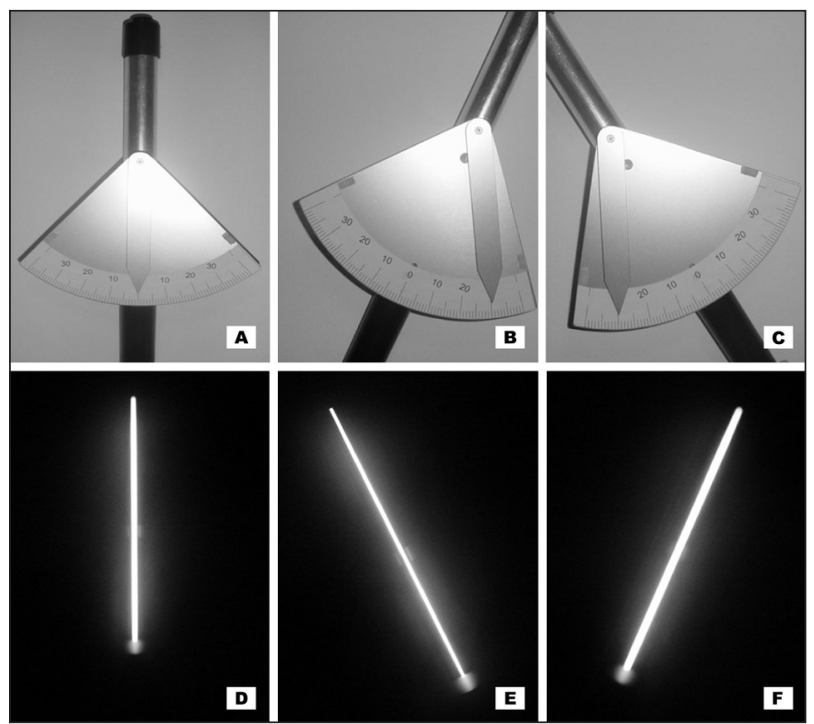

Fig 1. Aparelho para medida da VVS. A figura mostra como é realizada a medida em ângulos do ponto de vista do examinador ( $A, B$ e $C$ ), com o bastão na posição vertical $(A)$, quando o bastão é inclinado para esquerda (B) e para a direita (C) do indivíduo. Medida da VVS do ponto de vista do indivíduo que está sendo examinado, com o bastão na posição vertical $(D)$, inclinado para a esquerda (E), e para a direita $(F)$ do indivíduo. utilizado o aparelho portátil de menor precisão que os aparelhos computadorizados. Também não há estudos que avaliem os valores normais da VVS na população brasileira. Desta forma, os objetivos deste estudo foram: (1) avaliar a VVS utilizando um aparelho portátil; (2) estudar a VVS em indivíduos normais de uma amostra representativa da população brasileira, a fim de estabelecer a padronização dos valores normais para o método.

\section{MÉTODO}

Este estudo foi realizado com a aprovação da Comissão de Ética do Hospital das Clínicas da FMUSP e com a assinatura do Termo de Consentimento Livre e Esclarecido.

Foram avaliados 160 indivíduos normais, com distribuição considerada como representativa da população brasileira. A amostra foi dividida em faixas etárias de 10 anos, sendo a primeira menor que 30 anos e a última maior ou igual a 70 anos. Os critérios de exclusão foram: (1) antecedente de doença vestibular confirmada ou suspeita; (2) déficit auditivo unilateral ou bilateral; (3) distúrbio cognitivo; (4) alteração grave da acuidade visual (indivíduo não conta dedos); (5) antecedente de doença neurológica.

A medida da VVS foi realizada com indivíduo sentado na posição ortostática (vertical) a 1,5 $\mathrm{m}$ do examinador. A VVS foi determinada usando-se um bastão portátil, longo e luminoso (luz fluorescente) de $24 \mathrm{~cm}$ posicionado em frente ao indivíduo (Fig 1). O bastão foi colocado na altura dos olhos. O indivíduo usou óculos de lentes com filtro de luz que impossibilitava qualquer outra aferência visual, a não ser a luz fluorescente, percebida como uma linha verde. Foram realizados dez ajustes da VVS, um para cada lado totalizando cinco em cada direção de movimento (sentidos horário e anti-horário). De um ângulo de 40 graus uma vez para o lado esquerdo ou direito, o indivíduo foi solicitado a posicionar o bastão até a posição que Ihe parecesse vertical. Os ajustes foram feitos pelo examinador que seguiu as instruções verbais do indivíduo. As inclinações angulares da vertical foram medidas em graus e definidas como positivas para os desvios no sentido horário e negativas no sentido anti-horário em relação ao paciente. Com os resultados obtidos foi calculada a média de 10 ajustes do bastão.

$\mathrm{Na}$ análise estatística, o teste de Kruskal-Wallis ${ }^{10}$ foi usado para comparar as diferenças dos desvios da VVS entre os grupos etários. As diferenças entre os gêneros foram comparadas pelo teste de Mann-Withney. $O$ teste de Levene ${ }^{10}$ foi realizado para estudar as variâncias das inclinações da VVS nas diferentes faixas etárias. Foi aceito o nível de significância de $5 \%$.

\section{RESULTADOS}

Foram avaliados 74 homens e 86 mulheres entre as idades de 16 e 85 anos. Na análise descritiva, os resultados da VVS apresentaram valores entre $-2,0$ graus e $+2,4$ graus (média $+0,18$ ), com desvio padrão (DP) de 0,77. 
Tabela. Valores descritivos das medidas da VVS em relação aos grupos etários.

\begin{tabular}{lcccc}
\hline $\begin{array}{l}\text { Faixa etária } \\
\text { (anos) }\end{array}$ & $\mathrm{N}$ & Média & $\mathrm{DP}$ & $\mathrm{IC}$ \\
\hline$<30$ & 59 & 0,21 & 0,64 & 0,04 a 0,38 \\
30 a 39 & 28 & 0,14 & 0,67 & $-0,12$ a 0,40 \\
40 a 49 & 28 & 0,25 & 0,60 & 0,02 a 0,49 \\
50 a 59 & 24 & 0,06 & 1,04 & $-0,38$ a 0,50 \\
60 a 69 & 14 & $-0,11$ & 0,90 & $-0,63$ a 0,41 \\
$\geq 70$ & 07 & 0,67 & 1,17 & $-0,41$ a 1,75 \\
Total & 160 & 0,18 & 0,77 & 0,06 a 0,30 \\
\hline
\end{tabular}

Quando os resultados da VVS são analisados separadamente em relação ao gênero, a VVS no sexo feminino teve média de $+0,08^{\circ}$, com DP de 0,74 . No sexo masculino, a média foi $+0,29^{\circ}$ e o DP 0,79 . A Tabela mostra que os valores médios dos desvios da VVS não têm diferença com a idade $(p=0,40)$, porém os desvios padrões e os intervalos de confiança são maiores à medida que aumenta a faixa etária. $\mathrm{O}$ teste de Levene mostrou que a VVS teve inclinações crescentes com o aumento da faixa etária $(p=0,016)$. Os intervalos de normalidade, que foram calculados considerando que as medidas da VVS aumentam com a idade (Fig 2).

\section{DISCUSSÃO}

Neste estudo, a avaliação da VVS em 160 indivíduos normais mostrou que os desvios de julgamento da vertical verdadeira tiveram média de $+0,20^{\circ}$ e DP 0,78 . A utilização de um aparelho portátil para a medida da VVS tem como vantagem a possibilidade de realização do teste tanto em ambulatório como em pacientes internados, sendo de fácil transporte e manuseio. Friedmann descreveu que os controles tiveram uma média de erro do julgamento da vertical ou horizontal verdadeiras entre -2 e +2 graus $^{6}$. Dieterich e Brandt avaliaram a VVS em 110 indivíduos normais, com média $+0,3^{\circ}$ e desvio padrão da média de $\pm 0,9$ considerando a faixa de normalidade das inclinações da VVS entre $-0,6^{\circ}$ e $+1,2^{\circ 11}$. Nestes estudos, o teste da VVS foi realizado em aparelhos não portáteis.

A comparação dos desvios da VVS entre os indivíduos normais de diferentes grupos etários mostrou que as médias das inclinações da VVS não tiveram diferença com a idade, porém os intervalos de confiança foram maiores com o aumento da faixa etária. Este dado tem importância clínica porque, apesar das médias da VVS não mudarem com a idade, os indivíduos jovens têm inclinações da VVS dentro de um
Fig 2. Intervalos de normalidade da VVS.

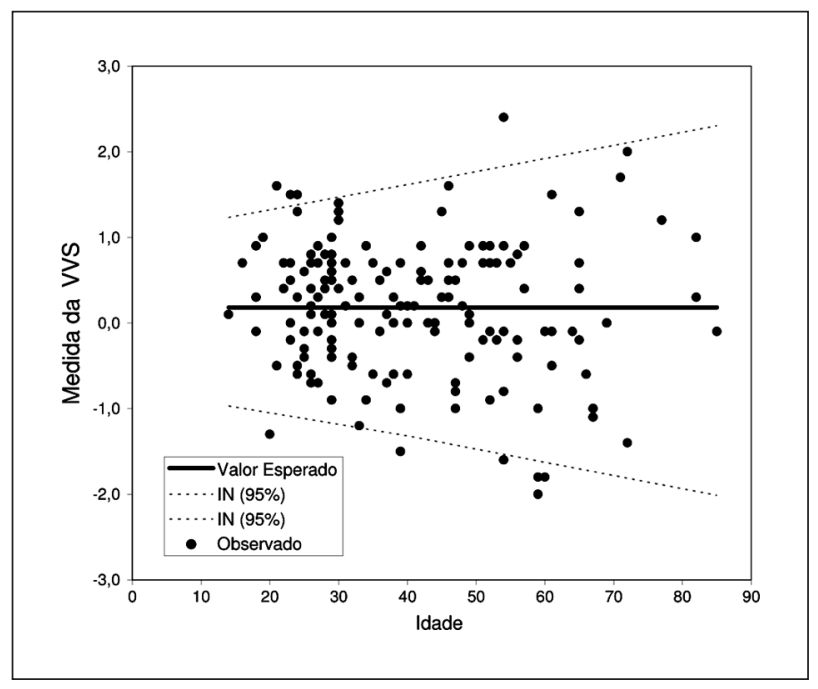

intervalo de normalidade menor que os mais idosos. Por exemplo, uma inclinação da VVS de $+1,5^{\circ}$ em uma pessoa de 25 anos será considerada acima do intervalo de normalidade para a faixa etária; entretanto em um indivíduo de 70, será considerada normal.

Kobayashi e cols demonstraram que as médias do julgamento da VVS quando feitas com o indivíduo na posição vertical e estática não sofreram alterações em relação à idade. No entanto, as variâncias dos intervalos de confiança nas diferentes faixas etárias não foram avaliadas nesse estudo. Quando a medida da VVS foi feita dinamicamente, ou seja, o ajuste do bastão luminoso na posição vertical é feito enquanto o plano de fundo gira no sentido horário e anti-horário, há um aumento gradual da inclinação da VVS nas faixas etárias maiores, o que poderia ser explicado pelo aumento da contribuição dos sinais visuais e diminuição da contribuição dos sinais vestibulares nos indivíduos com maior idade ${ }^{12}$. Dieterich e Brandt perceberam um erro padrão da média um pouco maior nos indivíduos acima de 60 anos, mas consideraram que a percepção da VVS permaneceu constante nas diferentes faixas etárias ${ }^{11}$.

Sintomas como perda de equilíbrio aumentam com a idade. Alguns estudos correlacionam esta queixa com o declínio da função dos sistemas vestibular, visual e músculo-esquelético relacionado ao enveIhecimento normal. O estudo longitudinal de Baloh mostrou decréscimo das respostas vestibulares em indivíduos idosos normais não associado a alterações patológicas do sistema vestibular ${ }^{13}$. O comprometimento da função vestibular idade-dependente está relacionado com a degeneração de células ciliadas e otólitos nos órgãos otolíticos e diminuição do nú- 
mero de neurônios no gânglio de Scarpa (gânglio vestibular) ${ }^{14,15}$. As alterações idade-dependentes do sistema vestibular em indivíduos normais poderiam estar relacionadas com a maior variância encontrada nas inclinações da VVS em indivíduos mais idosos.

O presente estudo mostrou que as inclinações da VVS não apresentaram diferença em relação ao gênero. Como esta comparação não foi descrita na literatura, acreditamos que nos estudos anteriores também não foram encontradas diferenças nos valores da VVS entre os gêneros.

Em conclusão, a função otolítica envolve a orientação estática gravitacional e movimentos com aceleração linear da cabeça, com consequente manutenção da postura e do equilíbrio. O teste da VVS avalia a percepção de verticalidade de um indivíduo. As inclinações da VVS são um sinal sensível da alteração do tono vestibular e ocorrem nas lesões vestibulares periféricas ou centrais em qualquer localização dentro das vias vestibulares, desde o labirinto até o córtex vestibular. A utilização deste método com um aparelho portátil em indivíduos normais mostrou que existe diferença entre os erros no julgamento da VVS em relação à idade.

\section{REFERÊNCIAS}

1. Kandel ER, Schwartz JH, Jessell TM. Principles of neural science. 4.Ed New York: Elsevier, 2000:801-831.

2. Brandt T. Vertigo, its multisensory syndromes. 2.Ed. London: Springer Verlag, 1999.

3. Kanashiro AMK, Pereira CB, Melo ACP, Scaff M. Diagnóstico e tratamento das principais síndromes vestibulares. Arq Neuropsiquiatr 2005;63:140-144.

4. Kanashiro AMK, Pereira CB, Barbosa ER, Scaff M. Avaliação da função vestibular através da vertical visual subjetiva. Rev Bras Neurol 2005;41:5-9.

5. Fetter M. Assessing vestibular function: which tests, when? J Neurol 2000;247:335-342.

6. Friedmann G. The judgment of the visual vertical and horizontal with peripheral and central vestibular lesions. Brain 1970;93:313-328.

7. Brandt T, Dieterich M, Danek A. Vestibular cortex lesions affect the perception of verticality. Ann Neurol 1994;35:403-412.

8. Brandt T, Dieterich M. Vestibular syndromes in the roll plane: topographic diagnosis from brainstem to cortex. Ann Neurol 1994;36:337347.

9. Herdman S. Reabilitação vestibular. 2.Ed. São Paulo: Manole, 2002: 192-211.

10. Neter J, Kutner MH, Nachtsheim CJ, Wasserman W. Applied linear statistical models. 4.Ed. Ilinois: Richard D. Irwing, 1996.

11. Dieterich M, Brandt T. Ocular torsion and tilt of subjective visual vertical are sensitive brainstem signs. Ann Neurol 1993;33:292-299.

12. Kobayashi H, Hayashi $Y$, Higashino K, et al. Dinamic and static subjective visual vertical with aging. Auris, Nasus, Larynx 2002;29:325-328.

13. Baloh RW, Enrietto J, Jacobson KM, Lin A. Age-related changes in vestibular function. A longitudinal study. Ann N Y Acad Sci 2001;942: 210-219.

14. Creasey H, Rapoport SI. The aging human brain. Ann Neurol 1985;17: 2-10.

15. Rauch SD, Velazquez-Villasenor L, Dimitri PS, Merchant SN. Decreasing hair cell counts in aging humans. Ann N Y Acad Sci 2001;942: 220-227. 